Western University

Scholarship@Western

Department of Economics Research Reports

Economics Working Papers Archive

1972

\title{
Another Look at Tests of Equality between Sets of Coefficients in Two Linear Regressions
}

Ronald L. Oaxaca

Follow this and additional works at: https://ir.lib.uwo.ca/economicsresrpt

Part of the Economics Commons

Citation of this paper:

Oaxaca, Ronald L.. "Another Look at Tests of Equality between Sets of Coefficients in Two Linear Regressions." Department of Economics Research Reports, 7204. London, ON: Department of Economics, University of Western Ontario (1972). 
Research Report 7204

\section{ANOTHER LOOK AT TESTS OF EQUALITY}

BETWEEN SETS OF COEFFICIENTS IN

TWO LINEAR REGRESSIONS

by

Rona1d L. Oaxaca

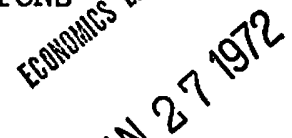

June, 1972 


\author{
Another Look at Tests of Equality \\ Between Sets of Coefficients in \\ Two Linear Regressions*
}

by

\title{
RONALD L. OAXACA
}

\section{Introduction}

Undoubtedly, anyone with at least an introductory course in econometrics has been exposed to the "Chow" test. As popularized by Johnston [4, pp. 136-138], the "Chow" test is a statistical means for deciding whether or not a linear regression run on one group of observations is significantly different from the same linear regression run on another group.

For some purposes it suffices to test only for the overall structural difference; however, there are many instances in which the researcher would like to know which regressors give rise to the structural difference. In certain other cases a researcher may have reason to include a subset of regressors for one group of observations that are not applicable to the other group. By virtue of the difference in specification, the overall structure is different between the two groups of observations. Yet we may be interested in structural differences with respect to the subset of regressors that the two linear regressions have in common.

The answers to these additional questions can be found in Chow's article on the subject [1], but their significance from an operational point of view is not immediately clear from the exposition. Recognizing the difficulty that some may have in following Chow's development of the testing procedures, F. M. Fisher has sought to simplify the development and illustrate the unity of Chow's tests with the $F$ test for the significance of subsets of

* This paper owes much to the conversations I have had with Orley Ashenfelter and Tom Valentine on this subject. 
coefficients in a single linear regression [2].

While all of this is familiar terrain to econometricians, it is not at all obvious to students nor to noneconometricians how one actually goes about implementing these tests. In so far as I know, there is no published source that clearly illustrates the operational manner in which one tests for differences in coefficients in the variety of situations previously discussed. It is therefore the purpose of this paper to present a straightforward, step by step, procedure for carrying out these tests. The emphas is is on ease of application and not on the actual derivation of the $F$ tests themselves. We shall be examining only those situations in which there are sufficient degrees of freedom to allow estimation of separate regressions for the two groups of observations.

2. Tests for Structural Differences When the Two Regressions are Identically Specified

We begin by postulating the following linear relationships:

$$
\begin{array}{ll}
y_{1 i}= & \sum_{j=1}^{k} \beta_{1 j} x_{1 i j}+e_{1 i}, \quad i=1, \ldots, n_{1} \\
y_{2 i}= & \sum_{j=1}^{k} \beta_{2 j} x_{2 i j}+e_{2 i}, \quad i=1, \ldots, n_{2}
\end{array}
$$

We assume that $e_{1}$ and $e_{2}$ have the same normal distribution. With the exception of the coefficients, we can drop the subscripts that denote which sample the observation is drawn from. The two linear relationships of (2.1) can then be combined into a single linear model:

$$
y_{i}=\sum_{j=1}^{k}\left[\beta_{1 j} x_{i j} D_{i}+\beta_{2 j} x_{i j}\left(1-D_{i}\right)\right]+e_{i}, \quad i=1, \ldots, n_{1}+n_{2}
$$

where $D_{i}=1$ if the observation is from group one, and

$$
=0 \text { otherwise. }
$$


Thus $D_{i}$ and $\left(1-D_{i}\right)$ are merely dummy variables that are interacted with all $k$ regressors. After collecting terms on $x_{i j} D_{i}$ in (2.2), we obtain

$$
y_{i}=\sum_{j=1}^{k}\left[\left(\beta_{1 j}-\beta_{2 j}\right) x_{i j} D_{i}+\beta_{2 j} x_{i j}\right]+e_{i}
$$

or

$$
\left.y_{i}=\sum_{j=1}^{k} r_{j} x_{i j} D_{i}+\beta_{2 j} x_{i j}\right]+e_{i}
$$

where $\Delta \beta_{\mathbf{j}} \equiv \beta_{1 \mathbf{j}}-\beta_{2 \mathbf{j}}$

With $2 k$ regressors and $n_{1}+n_{2}$ observations, equation (2.3) is estimated directly by ordinary least squares. The standard error and ' $t$ ' value corresponding to $\Delta \hat{\beta}_{j}$ tell us how significant the difference is between the coefficients if they had been estimated separately by ordinary least squares, i.e., $\hat{\beta}_{1 j}-\hat{\beta}_{2 j}$ for $j=1, \ldots, k$.

often one is interested in a joint test of significance for a subset of the $\Delta \hat{\beta}_{j}^{\prime} s$. For example, there may be some interest in testing for a structural difference with respect to a variable that is specified as a set of regressors. Suppose that the variable of interest consists of $k-h$ regressors, then the null hypothesis is that $\Delta \beta_{h+1}=\ldots=\Delta \beta_{k}=0$. In other words the nu1l hypothesis states that $y_{i}$ in (2.3) does not depend on $x_{i j} D_{i}, j=h+1, \ldots, k$. Following the standard procedure, we re-estimate equation (2.3) omitting those regressors whose coefficients are to be jointly tested for significance. The equation to be estimated is given by

$$
y_{i}=\sum_{j=1}^{h} \Delta \beta_{j}^{\prime} x_{i j} D_{i}+\sum_{j=1}^{k} \beta_{2 j}^{\prime} x_{i j}+e_{i}^{\prime}, \quad 0 \leq h<k
$$

Basically, the joint test of significance involves a comparison between the explained sums of squares for equations (2.3) and (2.4). 
Let $\operatorname{SST}=\sum_{i=1}^{\mathrm{n}_{1}+\mathrm{n}_{2}}\left(\mathrm{y}_{i}-\overline{\mathrm{y}}\right)^{2} \quad$ (Total sum of squares)

$$
\begin{array}{lr}
\text { SSR }=\sum_{i=1}^{n_{1}+n_{2}}\left(\hat{y}_{i}-\overline{\hat{y}}\right)^{2} & \begin{array}{r}
\text { (Explained sum of squares from the full } \\
\text { regression }(2.3)) \text { and, }
\end{array} \\
\mathrm{SSE}=\sum_{i=1}^{n_{1}+n_{2}} \hat{e}_{i}^{2} & \text { (Sum of squared residuals from (2.3)). }
\end{array}
$$

$\mathrm{SSR}^{\prime}$ and $\mathrm{SSE}^{\prime}$ are the corresponding statistics from (2.4), and $\Delta S S R=S S R-S^{\prime}$

On the hypothesis that $\Delta \beta_{h+1}=\ldots=\Delta \beta_{k}=0$, the ratio

$$
\frac{\Delta S S R /(k-h)}{S S E /\left(n_{1}+n_{2}-2 k\right)} \text { is distributed as } F_{n_{1}+n_{2}-2 k}^{2 k-h}
$$

High values of (2.5) lead to rejection of the null hypothesis. For a detailed development of this testing procedure see Goldberger [3, pp. 173-177] .

Some regression programs do not provide the explained sum of squares statistic in the computer print out; however, the F statistic can be computed from the $R^{2}$ and the sum of squared residuals. Given that

$$
\begin{aligned}
& \text { SST }=S S R+S S E \quad \text { and } \\
& R^{2}=\frac{S S R}{S S T}=1-\frac{S S E}{S S T} \quad \text { it follows that } \\
& \frac{R^{2}}{1-R^{2}}(S S E)=S S R . \quad \text { Thus }
\end{aligned}
$$

$$
\frac{\Delta S S R /(k-h)}{S S E /\left(n_{1}+n_{2}-2 k\right)}=\frac{\left[\begin{array}{lll}
\frac{R^{2}}{1-R^{2}}(S S E)-\frac{R^{2}}{1-R^{2^{\prime}}}\left(S S E^{\prime}\right)
\end{array}\right] /(k-h)}{S S E /\left(n_{1}+n_{2}-2 k\right)} \cdot
$$


In terms of the approach taken here, the "Chow" test described in Johnston [4, pp. 136-138;5, pp. 192-207] is equivalent to and nothing more than a joint test of all the $\Delta \beta_{j}{ }^{\prime} s, i . e ., h=0$. Under our procedures equation (2.3) is re-estimated omitting $x_{i j} D_{i}, j=1, \ldots, k$ and therefore becomes

$$
y_{i}=\sum_{j=1}^{k} \beta_{j} x_{i j}+\mu_{i}, \quad i=1, \ldots, n_{1}+n_{2}
$$

On the hypothesis that $\Delta \beta_{i}=\ldots=\Delta \beta_{k}=0$, the ratio

$$
\frac{\left(\mathrm{SSR}-\mathrm{SSR}^{\prime \prime}\right) / \mathrm{k}}{\mathrm{SSE} /\left(\mathrm{n}_{1}+\mathrm{n}_{2}-2 \mathrm{k}\right)} \quad \text { is distributed as } \mathrm{F}_{\mathrm{n}_{1}}^{\mathrm{k}}+\mathrm{n}_{2}-2 \mathrm{k}
$$

(where SSR" is the explained sum of squares corresponding to equation $(2.6))$.

In the appendix to this paper, a short proof is given that (2.7) is identical to the ratio that would be computed by following Johnston's steps for testing for an overall structural difference between two linear relations.

3. Tests for Structural Differences When the Two Regressions have Different Specifications

Testing for structural differences can be generalized to those cases in which not all of the regressors in the linear relations are the same between the two groups of observations. The general functional relationships may differ between the two groups as follows:

$$
y_{1}=f_{1}(x, W)+e_{1}
$$

$$
y_{2}=f_{2}(x, z)+e_{2}
$$

where $\mathrm{X}$ is a vector of regressors common to both groups, and $W$ and $\mathrm{Z}$ are vectors of regressors specific to groups one and two respectively 
The linear regression form of $(3.1)$ can be expressed as

$$
y_{1 i}=\sum_{j=1}^{k} \beta_{1 j} x_{1 i j}+\sum_{j=1}^{k} \alpha_{1 j} w_{1 i j}+e_{1 i}, \quad i=1, \ldots, n_{1}
$$

$$
y_{2 i}=\sum_{j=1}^{k} \beta_{2 j} x_{2 i j}+\sum_{j=1}^{k} \gamma_{2 j} z_{2 i j}+e_{2 i}, \quad i=1, \ldots, n_{2}
$$

Next, the two separate linear relations of (3.2) are combined into a single expression:

$$
\begin{aligned}
y_{i}= & \sum_{j=1}^{k} \bar{B}_{1 j} x_{i j} D_{i}+\beta_{2 j} x_{i j}\left(1-D_{i}\right)+\sum_{j=1}^{k} \alpha_{1 j} w_{i j} D_{i} \\
& +\sum_{j=1}^{k} \gamma_{2 j} z_{i j}\left(1-D_{i}\right)+e_{i}, \quad i=1, \ldots, n_{1}+n_{2}
\end{aligned}
$$

By rearranging the terms inside the first summation of (3.3), we obtain

$$
\left.y_{i}=\sum_{j=1}^{k} r \Delta \beta_{j} x_{i j} D_{i}+\beta_{2 j} x_{i j}\right\rceil+\sum_{j=1}^{k_{1}} \alpha_{1 j} w_{i j} D_{i}+\sum_{j=1}^{k_{2}} \gamma_{2 j} z_{i j}\left(1-D_{i}\right)+e_{i}
$$

With $2 k+k_{1}+k_{2}$ regressors and $n_{1}+n_{2}$ observations, equation (3.4) is estimated directly by ordinary least squares. The standard error and ' $t$ ' value corresponding to $\Delta \hat{\beta}_{j}$ tell us how significant the difference is between $\hat{\beta}_{1 j}$ and $\hat{\beta}_{2 j}$ had we estimated the two equations of (3.2) separately by ordinary least squares.

The joint test of significance for a subset of the $\Delta \hat{\beta}_{j}^{\prime}$ 's in (3.4) parallels the joint test described in section (2): Equation (3.4) is reestimated omitting those regressors whose coefficients are to be jointly tested for significance. of course we are only interested in a joint test from among the coefficients of the regressors that the two groups have in common. Suppose the $k-h$ regressors to be omitted from (3.4) are $x_{i j} D_{i}, j=h+1, \ldots, k$, then the null hypothes is is that $\Delta \beta_{h+1}=\ldots=\Delta \beta_{k}=0$. 
Accordingly the $F$ ratio for the joint test is

$$
\begin{aligned}
& \frac{\Delta \operatorname{SSR} /(k-h)}{\operatorname{SSE} /\left(\mathrm{n}_{1}+\mathrm{n}_{2}-2 \mathrm{k}-\mathrm{k}_{1}-\mathrm{k}_{2}\right.} \\
& \text { which is distributed as } \mathrm{F}_{\mathrm{n}_{1}+\mathrm{n}_{2}-2 k-\mathrm{k}_{1}-\mathrm{k}_{2}}^{\mathrm{k}-\mathrm{h}} \text {. }
\end{aligned}
$$

Although the linear relations for the two groups are specified differently, we find that we can still test for equality between the coefficients of the regressors common to the two linear relationships. Depending on the interests of the researcher, these tests can be made individually or jointly.

\section{Example}

An application of the testing procedures presented in sections (2) and (3) is illustrated by an example taken from a cross-section study of male-female wage differentials [6]. Separate wage regressions were estimated for the white males and white females in a household sample drawn from the 1967 Survey of Economic Opportunity. The natural logarithm of the hourly wage was the dependent variable. The independent variables are very briefly described here:

Experience, age less years of schooling completed less six years;

Education, years of schooling completed;

Health Problems, dummy variable $=1$ if the individual reports health problems that affect the kind or amount of work he can perform, and ' 0 ' otherwise; Part-Time, dummy variable $=1$ if the individual works less than thirty-five hours a week, and ' 0 ' otherwise;

Migration, dummy variable $=1$ if the individual has maintained a residence more than fifty miles from his current address since the age of seventeen, and ' 0 ' otherwise; 
YRSM, number of years since the individual last migrated;

Spouse Present, Spouse Absent, Widowed, Divorced, dummy variables for marital status with Never Married as the reference group; SMSA 250, SMSA 500, SMSA 750, SMSA 750t, dummy variables for size of urban area where the Standard Metropolitan Statistical Area is less than 250,000, or greater than or equal to 250,000 but less than 500,000 , or greater than or equal to 500,000 but less than 750,000 , or greater than or equal to 750,000 , with Urban, Non SMSA as the reference group;

North East, North Central, West, dummy variables for the census region with South as the reference group; and Children, number of children the female worker has given birth to. The Experience, Education, and $\underline{\text { YRSM }}$ variables also include quadratic terms. The separate wage equations are given by

$$
\ln \left(w_{f i}\right)=\sum_{j=1}^{21} \beta_{f j} x_{f i j}+\alpha_{f} w_{f i}+e_{f i}, \quad i=1, \ldots, 4,962
$$

$(4.1)$

$$
\ln \left(w_{m i}\right)=\sum_{j=1}^{21} \beta_{m j} x_{m i j} \quad+e_{m i}, \quad i=1, \ldots, 8,123
$$

where $w_{f i}$ represents the children variable and the subscripts ' $m$ ' and ' $\mathrm{f}$ ' denote males and females respectively.

Except for the children variable occurring only in the female wage equation, the two equations are identically specified. The estimated differences between the coefficients of the regressors common to both equations are directly obtained from the combined regression equation

$$
\begin{array}{r}
\ln \left(w_{i}\right)=\sum_{j=1}^{21}-\Delta \beta_{j} x_{i j} D_{i}+\beta_{m j} x_{i j} j+\alpha_{f} w_{i} D_{i}+e_{i}, \\
i=1, \ldots, 13,085
\end{array}
$$




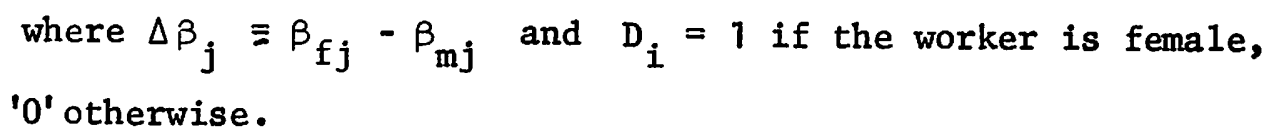

The results for both the separate and combined regressions are presented in Table (4.1). Since the estimated parameters of the separate regression equations of $(4.1)$ are all contained in the combined regression (4.2), it may seem redundant to repeat them; however, they are repeated in Table (4.1) for illustrative purposes only. Although the variances of the true residuals corresponding to the two groups may be the same, in practice the standard error of estimate is seldom identical for separate regressions run on two groups of observations. Consequently, the ' $t$ ' values and implied standard errors from the combined regression in Table (4.1) differ somewhat from the values obtained through the separate regressions. This of course presents no problem when one considers that the standard errors from the combined regression are based on more information than in the separate regressions.

Aside from the children variable, are the wage equations for males and females significantly different? The answer to this question requires a joint test of significance for differences in the coefficients of the twenty-one regressors common to both groups o Consequently, equation (4.2) is re-estimated omitting $x_{i j} D_{i}, j=1, \ldots, 21$. The resultant equation is (4.3) $\quad \ln \left(w_{i}\right)=\sum_{j=1}^{21} \beta_{j} x_{i j}+\alpha_{f}^{\prime} w_{i} D_{i}+e_{i}^{\prime}, \quad i=1, \ldots, 13,085$

The pertinent information for the $F$ test is provided in Table (4.2). 


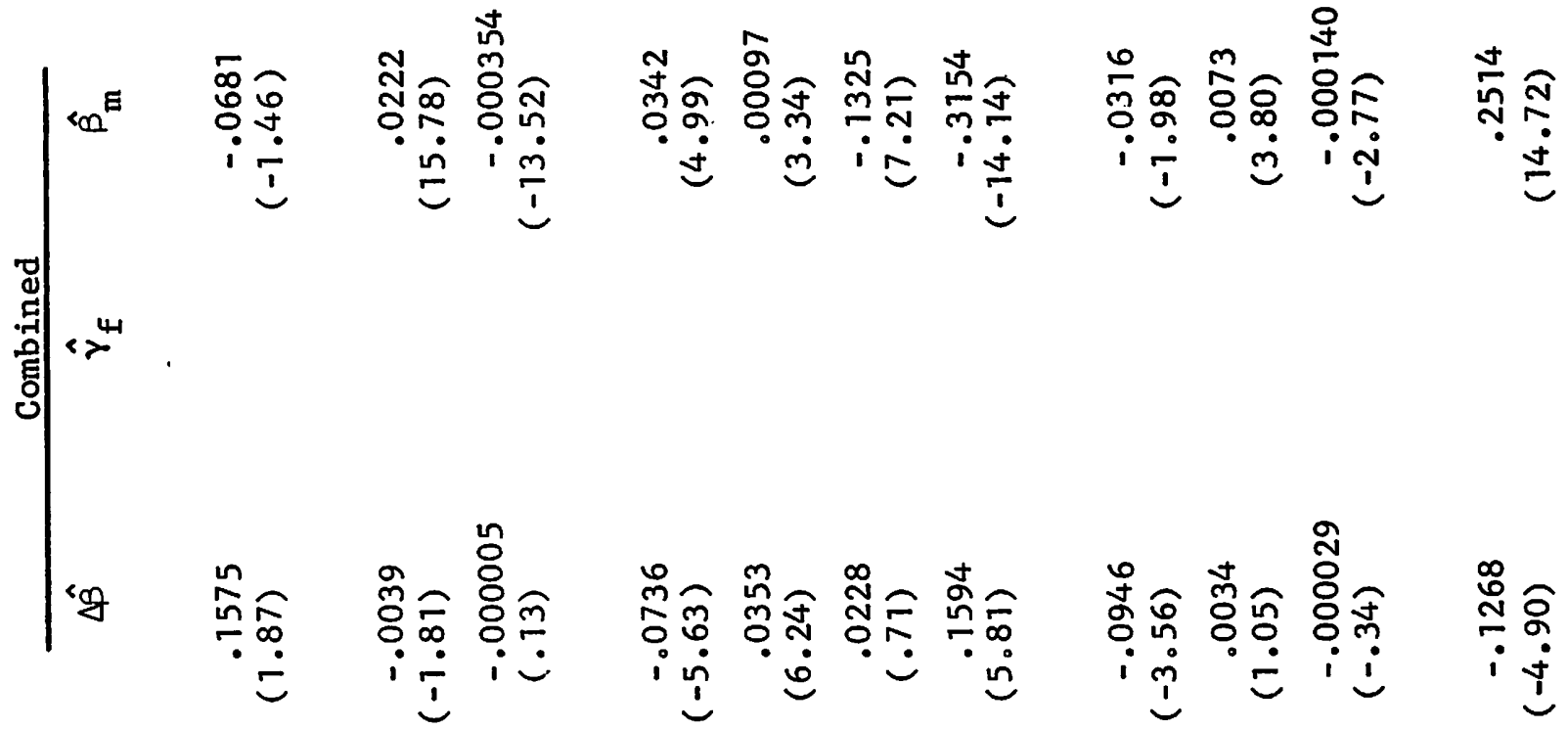

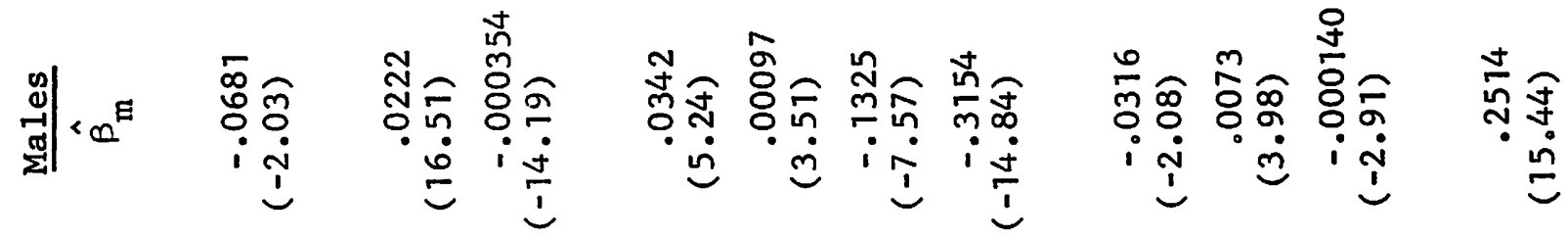

年

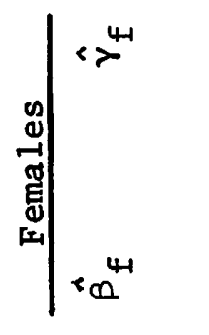

ัૅ

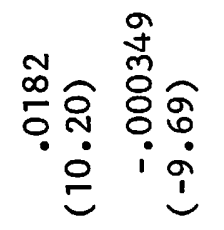

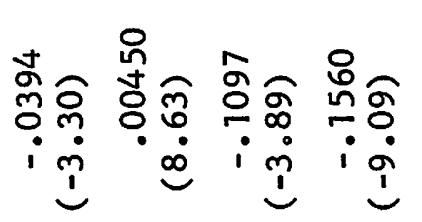

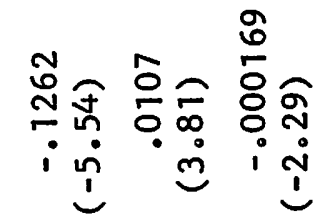

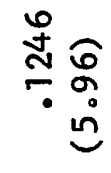
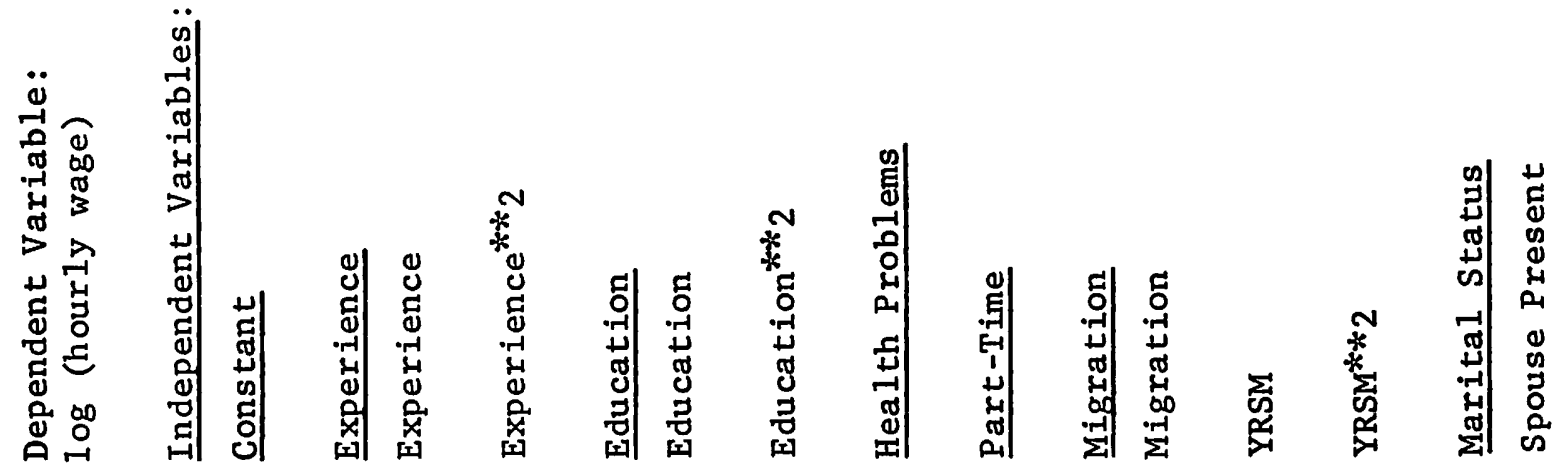


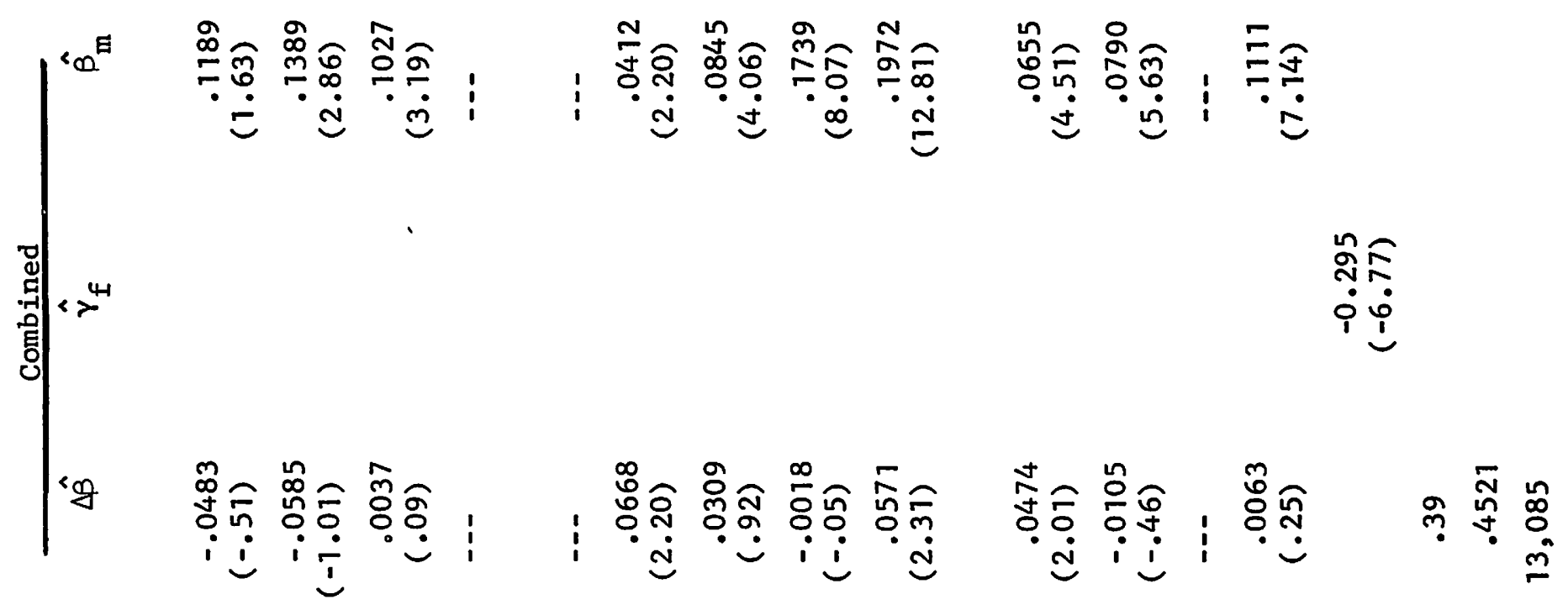

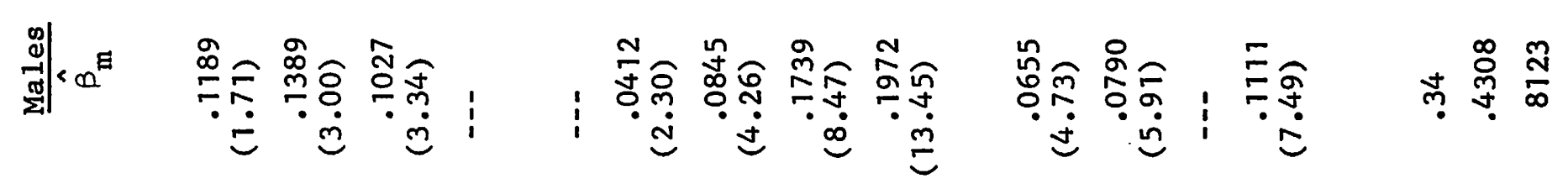

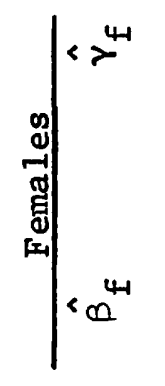

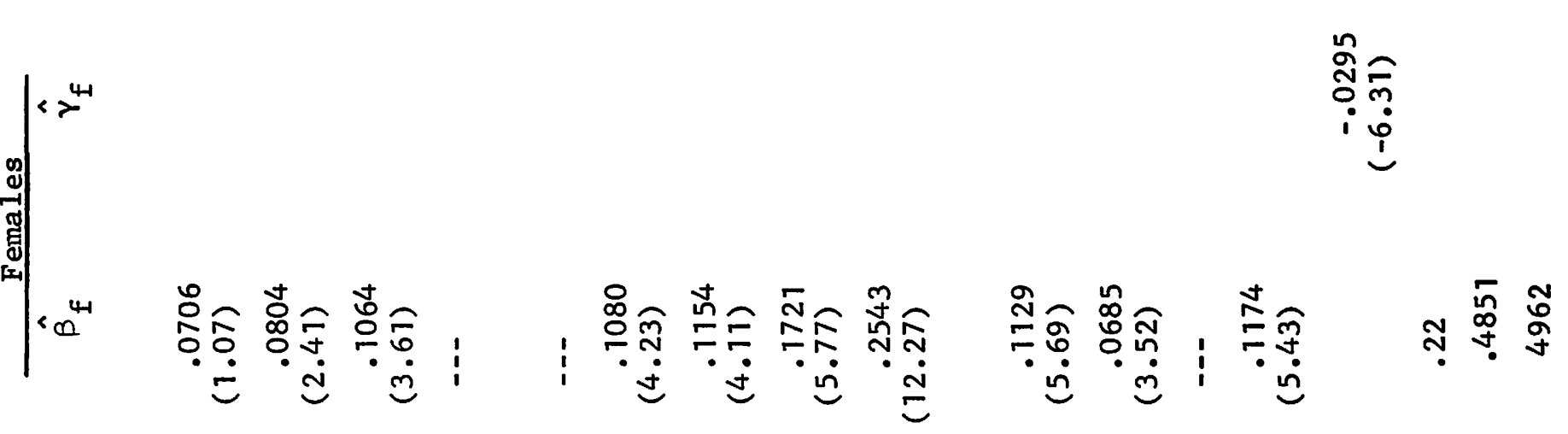

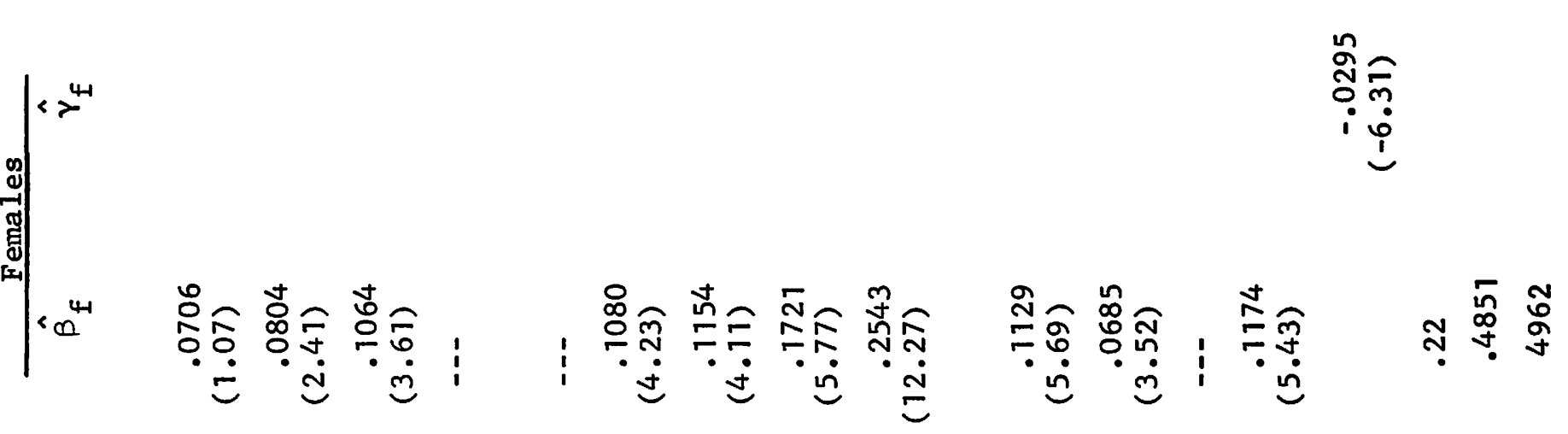

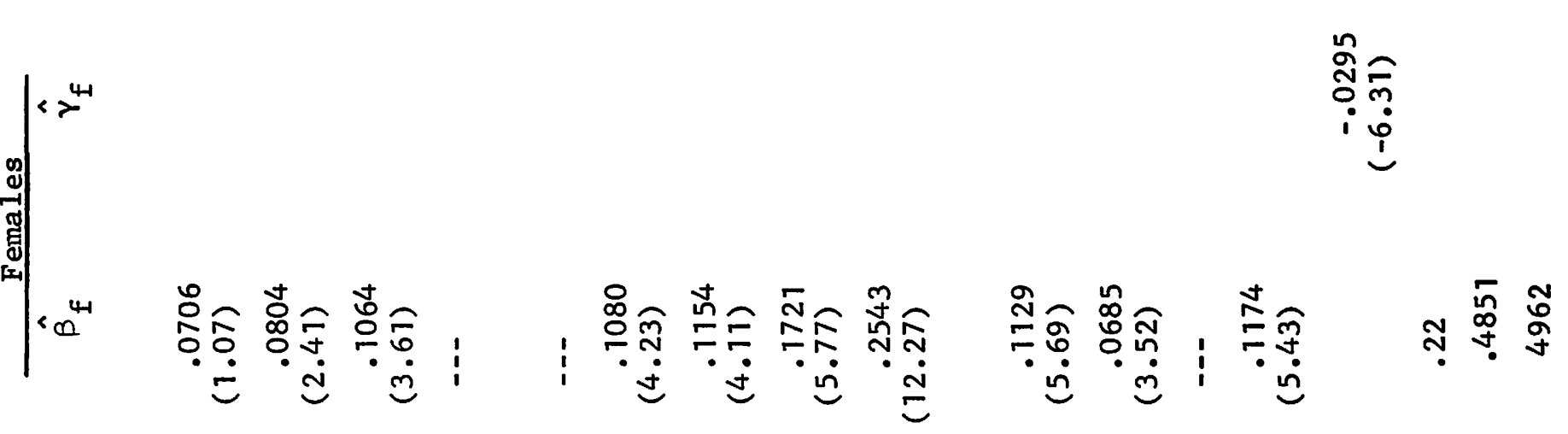

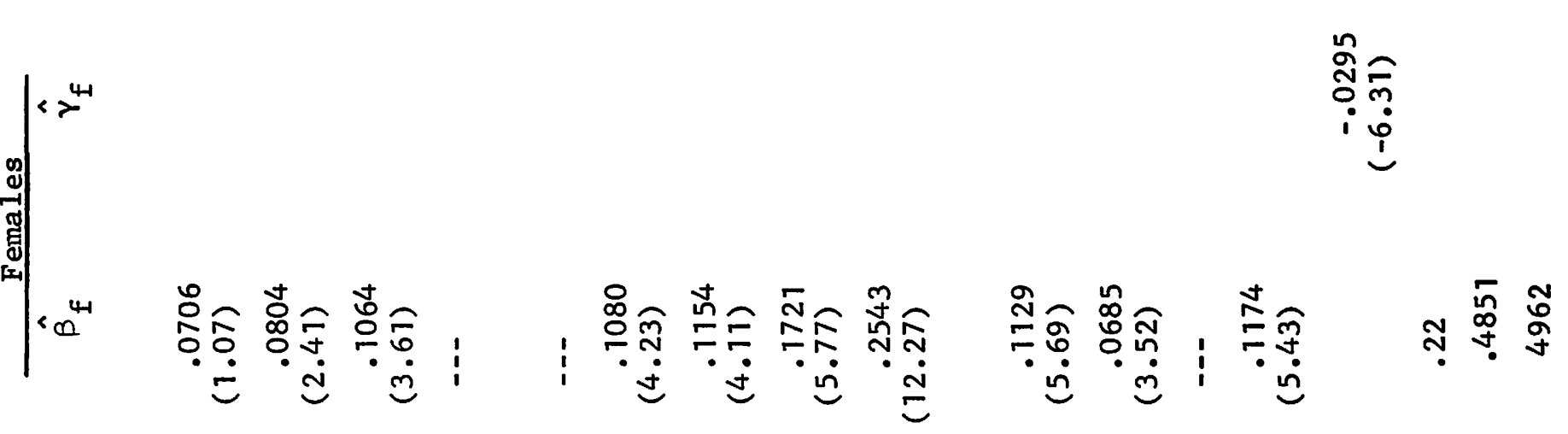

.

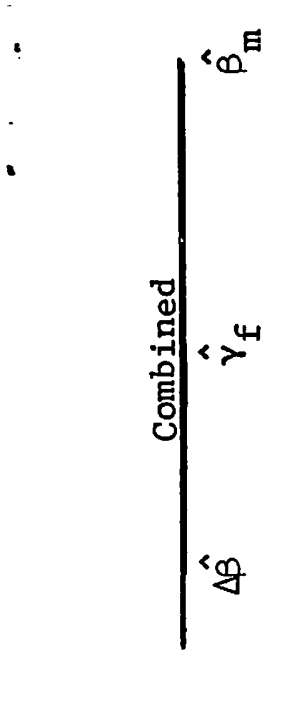

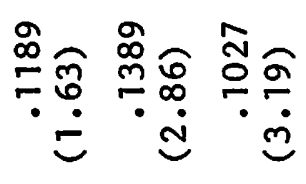

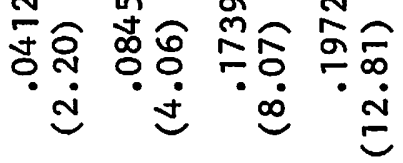

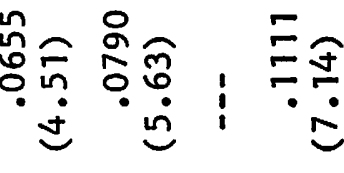

กิ๊ี

훙

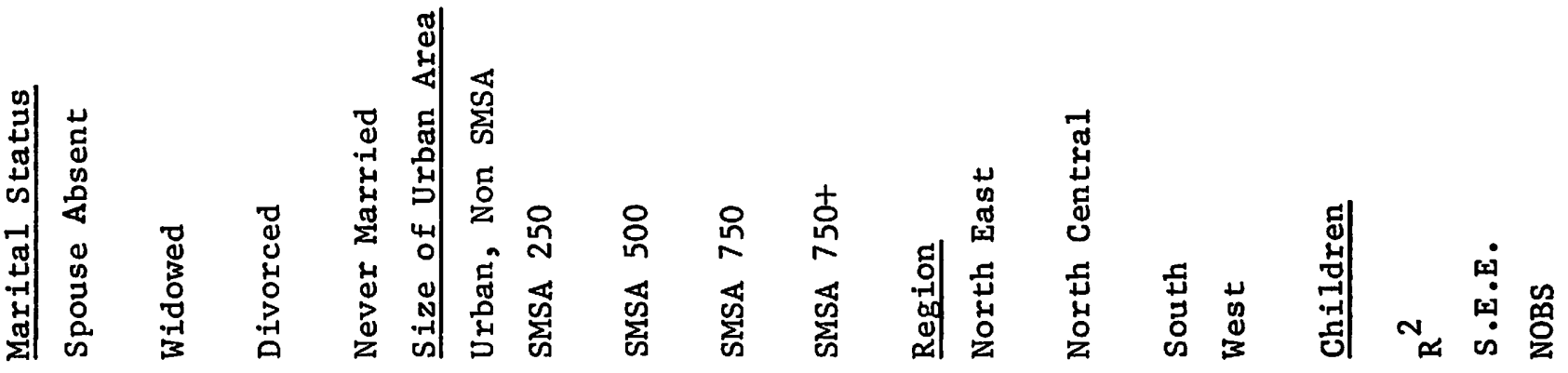


TABLE 4.2

\begin{tabular}{c|c|c}
\hline Regression & SSR & SSE \\
\hline$(4.2)$ & 1696.7331 & 2665.9758 \\
$(4.3)$ & 1503.3711 & \\
\hline
\end{tabular}

Using formula (3.5) with $h=k_{2}=0$, the $F$ ratio is

$$
\begin{aligned}
\frac{\Delta S S R / 21}{S S E / 13,042} & =\frac{193.3620 / 21}{2665.9758 / 13,042} \\
& =\frac{9.2077}{.2044} \\
& =45.05
\end{aligned}
$$

In order for the differences in the coefficients to be jointly significant at the $1 \%$ level, the $\mathrm{F}$ ratio must exceed 1.88 ; therefore, we can easily reject the null hypothesis that the wage equations are the same for males and females with respect to the regressors common to both.

\section{Summary}

Beginning with identical linear specifications for two groups of observations, we briefly list the steps for testing for equality between sets of coefficients in the two linear relations:

1. Pool the two groups of observations $\left(n_{1}+n_{2}\right)$ and generate an additional set of regressors by interacting the original set $\left(x_{i j}\right)$ with a dummy variable that distinguishes between the two groups $\left(D_{i}\right)$.

2. Estimate the combined regression with $2 \mathrm{k}$ regressors by ordinary least squares.

3. The estimated coefficients $\left(\Delta \hat{\beta}_{j}\right)$ of the interaction terms $\left(X_{i j} D_{i}\right)$ 
are the estimated differences in the coefficients that could have been obtained by separately estimating the regressions for the two groups and then subtracting the estimated coefficients of one group from the estimated coefficients of the other, i.e., $\Delta \hat{\beta}_{j} \equiv \hat{\beta}_{1 j}-\hat{\beta}_{2 j}$. The corresponding ' $t$ ' values indicate the statistical significance of these differences.

4. The estimated coefficients $\left(\hat{\beta}_{2 j}\right)$ of the noninteracted terms $\left(x_{i j}\right)$ are identical to the estimated coefficients that would be obtained from a separate regression run for one of the two groups.

5. To perform a joint test for differences between a subset of coefficients $\Delta \hat{\beta}_{j}, j=h+1, \ldots, k$, re-estimate the combined regression omitting the $k-h$ regressors $x_{i h+1} D_{i}, \ldots, x_{i k} D_{i}$. The change in the explained sum of squares ( $\triangle S S R$ ) is the basis for computing the $F$ statistic

$$
\frac{\Delta S S R /(k-h)}{\operatorname{SSE} /\left(n_{1}+n_{2}-2 k\right.} \sim F_{n_{1}+n_{2}-2 k}^{k-h} \text {. }
$$

The standard "Chow" test corresponds to the case when $h=0$ (or $\mathrm{k}-\mathrm{h}=\mathrm{k}$ ).

Only slight changes to the above are required when the linear relations for the two groups are specified such that they have a subset of regressors in common and differ with respect to other regressors:

1. The combined regression will now include the regressors specific to each group. These are interacted with the dummy variable that represents the relevant group, i.e., $D_{i}$ or $\left(1-D_{i}\right)$.

2. The F statistic for joint tests becomes

$$
\frac{\Delta S S R /(k-h)}{\operatorname{SSE} /\left(n_{1}+n_{2}-2 k-k_{1}-k_{2}\right)}
$$


Hopefully, this paper has provided a simplified procedure for testing for equality between sets of coefficients in two linear regressions. Although the example in section (4) was taken from a cross section study, our testing procedures obviously apply to time series: One may be interested in testing whether the same linear relation applies to two different time periods. For another example of a time series application see [7]. 
Appendix

In this section we will prove that ordinary least squares estimation of the combined regression for two groups of observations is equivalent to ordinary least squares estimation of the separate regressions. We will also show that our test statistic for the overall structural difference between the coefficients in two linear relations is identical to the test statistic developed in Johnston [4, p. 137; 5, p. 199]

The development of the first proof is greatly facilitated by casting the model in matrix form:

$$
y_{1}=x_{1} \beta_{1}+e_{1}
$$

(A.1)

$$
y_{2}=x_{2} \beta_{2}+e_{2}
$$

where $y_{1}$ and $y_{2}$ are $n_{1} \times 1$ and $n_{2} \times 1$ vectors respectively;

$\mathrm{x}_{1}$ and $\mathrm{x}_{2}$ are $\mathrm{n}_{1} \times \mathrm{k}$ and $\mathrm{n}_{2} \times \mathrm{k}$ matrices respectively;

$\beta_{1}$ and $\beta_{2}$ are $k \times 1$ vectors; and

$e_{1}$ and $e_{2}$ are $n_{1} \times 1$ and $n_{2} \times 1$ vectors respectively.

It 1 s assumed that $e_{1}$ and $e_{2}$ have the same normal distribution. Through the appropriate use of dummy variables that identify which group the observation is drawn from, the two linear relationships of A.1) can be combined into a single linear model:

(A.2) $\left[\begin{array}{l}y_{1} \\ y_{2}\end{array}\right]=\left[\begin{array}{ll}x_{1} & 0 \\ 0 & x_{2}\end{array}\right]\left[\begin{array}{l}\beta_{1} \\ \beta_{2}\end{array}\right]+\left[\begin{array}{l}e_{1} \\ e_{2}\end{array}\right]$

or equivalently, 
(A.3)

$$
\begin{aligned}
& {\left[\begin{array}{l}
y_{1} \\
y_{2}
\end{array}\right]=\left[\begin{array}{l}
x_{1} \\
0
\end{array}\right] \beta_{1}+\left[\begin{array}{l}
0 \\
x_{2}
\end{array}\right] \beta_{2}+\left[\begin{array}{l}
e_{1} \\
e_{2}
\end{array}\right]} \\
& \text { Define } \Delta \beta \equiv \beta_{1}-\beta_{2} \text {, then } \beta_{1} \equiv \Delta \beta+\beta_{2} \\
& \text { Substituting for } \beta_{1} \text { in (A.3) we obtain } \\
& {\left[\begin{array}{l}
y_{1} \\
y_{2}
\end{array}\right]=\left[\begin{array}{l}
x_{1} \\
0
\end{array}\right] \Delta \beta+\left[\begin{array}{l}
x_{1} \\
x_{2}
\end{array}\right] \beta_{2}+\left[\begin{array}{l}
e_{1} \\
e_{2}
\end{array}\right]}
\end{aligned}
$$

or

(A.4)

$$
\begin{aligned}
& {\left[\begin{array}{l}
y_{1} \\
y_{2}
\end{array}\right]=\left[\begin{array}{ll}
x_{1} & x_{1} \\
0 & x_{2}
\end{array}\right]\left[\begin{array}{l}
\Delta \beta \\
\beta_{2}
\end{array}\right]+\left[\begin{array}{l}
e_{1} \\
e_{2}
\end{array}\right]} \\
& n_{1}+n_{2} \times 1 \quad n_{1}+n_{2} \times 2 k \quad 2 k \times 1 \quad n_{1}+n_{2} \times 1
\end{aligned}
$$

The linear model (A.4) is of course the matrix form of equation (2.3). We wish to show that the ordinary least squares estimator of $\Delta \beta$ in (A.4) is identical to the difference between the ordinary least squares estimators of $\beta_{1}$ and $\beta_{2}$ in the separate linear relationships of (A.1). We also wish to prove that the ordinary least squares estimators of $\beta_{2}$ in the combined regression of (A.4) and in the separate regression of (A.1) are identical. From ordinary least squares estimation of (A.4) we have

$$
\begin{aligned}
{\left[\begin{array}{l}
\Delta \hat{\beta} \\
\hat{\beta}_{2}
\end{array}\right] } & =\left[\left(\begin{array}{ll}
x_{1}^{\prime} & 0^{\prime} \\
x_{1}^{\prime} & x_{2}^{\prime}
\end{array}\right)\left(\begin{array}{ll}
x_{1} & x_{1} \\
0 & x_{2}
\end{array}\right)\right]^{-1}\left[\begin{array}{ll}
x_{1}^{\prime} & 0^{\prime} \\
x_{1}^{\prime} & x_{2}^{\prime}
\end{array}\right]\left[\begin{array}{l}
y_{1} \\
y_{2}
\end{array}\right] \\
& =\left[\begin{array}{ll}
x_{1}^{\prime} x_{1} & x_{1}^{\prime} x_{1} \\
x_{1}^{\prime} x_{1} & x_{1}^{\prime} x_{1}+x_{2}^{\prime} x_{2}
\end{array}\right]^{-1}\left[\begin{array}{l}
x_{1}^{\prime} y_{1} \\
x_{1}^{\prime} y_{1}+x_{2}^{\prime} y_{2}
\end{array}\right]
\end{aligned}
$$


Through the application of partitioned matrix inversion we obtain

$$
\begin{aligned}
{\left[\begin{array}{l}
\Delta \hat{\beta} \\
\hat{\beta}
\end{array}\right]=\left[\begin{array}{rr}
\left(x_{1}^{\prime} x_{1}\right)^{-1}+\left(x_{2}^{\prime} x_{2}\right)^{-1} & -\left(x_{2}^{\prime} x_{2}\right)^{-1} \\
-\left(x_{2}^{\prime} x_{2}\right)^{-1} & \left(x_{2}^{\prime} x_{2}\right)^{-1}
\end{array}\right]\left[\begin{array}{l}
x_{1}^{\prime} y_{1} \\
x_{1}^{\prime} y_{1}+x_{2}^{\prime} y_{2}
\end{array}\right] } \\
=\left[\begin{array}{c}
\left(x_{1}^{\prime} x_{1}\right)^{-1} x_{1}^{\prime} y_{1}-\left(x_{2}^{\prime} x_{2}\right)^{-1} x_{2}^{\prime} y_{2} \\
\left(x_{2}^{\prime} x_{2}\right)^{-1} x_{2}^{\prime} y_{2}
\end{array}\right]
\end{aligned}
$$

and thus

$$
\begin{aligned}
\Delta \hat{\beta} & =\left(x_{1}^{\prime} x_{1}\right)^{-1} x_{1} y_{1}-\left(x_{2}^{\prime} x_{2}\right)^{-1} x_{2}^{\prime} y_{2}=\hat{\beta}_{1}-\hat{\beta}_{2} \\
\hat{\beta}_{2} & =\left(x_{2}^{\prime} x_{2}\right)^{-1} x_{2}^{\prime} y_{2} .
\end{aligned}
$$

Using our notation, the $F$ statistic presented in Johnston [4, p. 137] for testing for overall differences between two linear regressions is

(A.5)

$$
\frac{\left[\mathrm{SSE}^{\prime \prime}-\left(\mathrm{SSE}_{1}+\mathrm{SSE}_{2}\right)\right] / \mathrm{k}}{\left(\mathrm{SSE}_{1}+\mathrm{SSE}_{2}\right) /\left(\mathrm{n}_{1}+\mathrm{n}_{2}-2 \mathrm{k}\right)}
$$

where SSE" is the sum of squared residuals from the pooled regression run without the interaction terms, i.e.,

$$
\left[\begin{array}{l}
y_{1} \\
y_{2}
\end{array}\right]=\left[\begin{array}{l}
x_{1} \\
x_{2}
\end{array}\right] \beta+\mu \text { as equation (2.6), }
$$

and $\mathrm{SSE}_{1}, \mathrm{SSE}_{2}$ are the sums of squared residuals from the separate regressions for groups one and two respectively.

In his second edition, Johnston develops the testing procedures in terms of the analysis of covariance [5, pp. 192-207]. As such, the analysis handles more than two groups of observations, but the sample size is assumed to be the same for all groups. The analysis is easily adapted to the present case of two groups with their sample sizes not necessarily the same. Also in the 
second edition, $\mathrm{SSE}_{1}+\mathrm{SSE}_{2}$ in the denominator of (A.5) was replaced by the identical sum of squared residuals from a combined regression of the form given in (A.2) [5, p. 199]. These are of course identical to the sum of squared residuals (SSE) from our combined regression (A.4). Replacing $\mathrm{SSE}_{1}+\mathrm{SSE}_{2}$ in (A.5) by SSE, we have

$$
\frac{(S S E \prime \prime}{S S E /\left(n_{1}+n_{2}-2 k\right)}
$$

Now SSE" = SST - SSR" and SSE = SST - SSR so that SSE" - SSE = SSR - SSR" . Therefore (A.5) is identical to

$$
\frac{\left(\mathrm{SSR}-\mathrm{SSR}^{\prime \prime}\right) / \mathrm{k}}{\mathrm{SSE} /\left(\mathrm{n}_{1}+\mathfrak{n}_{2}-2 \mathrm{k}\right)} \text { which is of course formula (2.7). }
$$

When the linear relationships for the two groups have different specifications but with a subset of regressors in common, the model in matrix form can be expressed as

$$
\mathrm{y}_{1}=\mathrm{x}_{1} \beta_{1}+\mathrm{w}_{1} \alpha_{1}+\mathrm{e}_{1}
$$

(A.6)

$$
y_{2}=x_{2} \beta_{2}+z_{2} \gamma_{2}+e_{2}
$$

where $y_{1}, y_{2}, x_{1}, x_{2}, \beta_{1}, \beta_{2}, e_{1}$, and $e_{2}$ have the same dimensions as in (A.1);

$\mathrm{W}_{1}$ and $\mathrm{z}_{2}$ are $\mathrm{n}_{1} \times \mathrm{k}_{1}$ and $\mathrm{n}_{2} \times \mathrm{k}_{2}$ matrices respectively; and $\alpha_{1}$ and $\gamma_{2}$ are $k_{1} \times 1$ and $k_{2} \times 1$ vectors respectively.

Combining the two linear relations of (A.6) into a single linear model, we have

(A.7) $\quad\left[\begin{array}{l}y_{1} \\ y_{2}\end{array}\right]=\left[\begin{array}{cccc}x_{1} & w_{1} & 0 & 0 \\ 0 & 0 & x_{2} & z_{2}\end{array}\right]\left[\begin{array}{l}\beta_{1} \\ \alpha_{1} \\ \beta_{2} \\ \gamma_{2}\end{array}\right]+\left[\begin{array}{l}e_{1} \\ e_{2}\end{array}\right]$ 
or

(A.8) $\quad\left[\begin{array}{l}y_{1} \\ y_{2}\end{array}\right]=\left[\begin{array}{l}x_{1} \\ 0\end{array}\right] \beta_{1}+\left[\begin{array}{c}w_{1} \\ 0\end{array}\right] \alpha_{1}+\left[\begin{array}{c}0 \\ x_{2}\end{array}\right] \beta_{2}+\left[\begin{array}{c}0 \\ z_{2}\end{array}\right] \gamma_{2}+\left[\begin{array}{l}e_{1} \\ e_{2}\end{array}\right]$.

Substituting $\Delta \beta+\beta_{2}$ for $\beta_{1}$ in (A.8), we obtain

$\left[\begin{array}{l}y_{1} \\ y_{2}\end{array}\right]=\left[\begin{array}{l}x_{1} \\ 0\end{array}\right] \Delta \beta+\left[\begin{array}{l}x_{1} \\ x_{2}\end{array}\right] \beta_{2}+\left[\begin{array}{l}w_{1} \\ 0\end{array}\right] \alpha_{1}+\left[\begin{array}{l}0 \\ z_{2}\end{array}\right] \gamma_{2}+\left[\begin{array}{l}e_{1} \\ e_{2}\end{array}\right]$

or

(A.9) $\left[\begin{array}{l}y_{1} \\ y_{2}\end{array}\right]=\left[\begin{array}{cccc}x_{1} & x_{1} & w_{1} & 0 \\ 0 & x_{2} & 0 & z_{2}\end{array}\right]\left[\begin{array}{l}\Delta \beta \\ \beta_{2} \\ \alpha_{1} \\ \gamma_{2}\end{array}\right]+\left[\begin{array}{l}e_{1} \\ e_{2}\end{array}\right]$

$$
\mathrm{n}_{1}+\mathrm{n}_{2} \times 1 \quad \mathrm{n}_{1}+\mathrm{n}_{2} \times 2 \mathrm{k}+\mathrm{k}_{1}+\mathrm{k}_{2} \underset{+\mathrm{k}_{2} \times 1}{2 \mathrm{k}+\mathrm{k}_{1}}+\mathrm{n}_{1}+\mathrm{n}_{2} \times 1
$$

It is a straightforward exercise to show that ordinary least squares estimation of $\Delta \beta_{1} \beta_{2}, \alpha_{1}$ and $\gamma_{2}$ in the combined regression of (A.9) is identical to ordinary least squares estimation of the separate linear relationships in (A.6); therefore, the proof is omitted. 


\section{References}

[1] Chow, Gregory C., "Tests of Equality Between Subsets of Coefficients in Two Linear Regressions," Econometrica, Vo1. 28, No. 3 (July, 1960), pp. 591-605.

[2] Fisher, Franklin M., "Tests of Equality Between Sets of Coefficients in Two Linear Regressions: an Expository Note," Econometrica, Vo1. 38, No. 2 (March, 1970), pp. 361-366.

[3] Goldberger, Arthur S., Econometric Theory, New York, John Wiley and Sons, 1964 .

[4] Johnston, J., Econometric Methods, New York, McGraw-Hi11 Book Company, Inc., 1963.

[5] Johnston, J., Econometric Methods, New York, McGraw-Hill Book Company, Inc., second edition, 1972.

[6] Oaxaca, Ronald L., "Male-Female Wage Differentials in Urban Labor Markets," unpublished Ph.D. dissertation, Princeton University, 1971.

[7] Valentine, Thomas J., "The Chow Test and Autocorrelation: A Comment," mimeographed paper, Department of Economics, University of Western Ontario, London, Canada, 1971. 